\title{
Use of additive technologies to increase effectiveness of design and use of a vacuum gripping devices for flexible containers
}

\author{
Alexey M. Makarov ${ }^{1, *}$, Oleg V. Mushkin ${ }^{1}$, and Maksim A. Lapikov ${ }^{1}$ \\ ${ }^{1}$ VSTU, Russia, 400005, Volgograd, pr. Lenina, 28
}

\begin{abstract}
In an effort to increase efficiency of the process of handling of an air-penetrating flexible container during its' filling with free-flowing materials a devise has been designed for automatic gripping, opening, holding and sealing of flexible container, containing vacuum gripping devise, pneumatic drive for its' transfer and a control system based on programmable logical controller. Vacuum gripping device can be produced using the method of additive technologies. It has a modular structure, while each module contains random or structured vacuum chambers located on work surface area, connected to a system of internally branched channels with the source of vacuum. Large number of small vacuum chambers in place allows to minimize leakage of air though pores and openings on the surface of the mouth of weaved flexible container, which increases reliability of holding and reduces energy consumption during the process of handling air-penetrating packaging during its' filling.
\end{abstract}

\section{Introduction}

In a modern automated packaging production, it is necessary to introduce robotic and mechatronic systems, allowing to fully automate the packaging process, thereby increasing the quality of the process, productivity and environmental friendliness, eliminating the human factor. An important task in the packaging process is manipulating of filling containers, especially in the case of air-penetrating flexible containers (FC). The task includes a complex of operations: gripping of FC from stack; opening and holding of FC during filling, transfer of filled FC to conveyer belt for its' stitching and transportation.

Various gripping devices can be used to solve the problem of automatic manipulation of FC from air-penetrating materials, including vacuum gripping devices (VGD), description of their designs, results of mathematical and computer modelling of their operation are presented in [1-5].

The device [5] allows to conduct a full cycle of operations of handling FC in sorting free-flowing products (FP) in automatic mode. However, if the flexible container is made from air-penetrating material, the effectiveness of its gripping, opening and holding during filling is reduced, since significant air leaks occur when the vacuum chambers of a sufficiently large size interact with the material of the flexible container. This reduces

*Corresponding author: amm34@mail.ru 
energy efficiency and reliability of gripping and holding a flexible container during transportation and filling, and increases the likelihood of incorrect opening and holding of container, which can lead to defects during packaging, partial loss of bulk material, increase dust generation and also decrease performance of the overall process. Existing VGDs, for example, described in [6-7], also do not allow to solve this problem.

\section{Materials and methods of research}

\subsection{Description of the device design}

A modular gripping device that allows to manipulate flexible containers has been developed to increase process efficiency of handling flexible container from air-penetrating material. (Fig. 1).

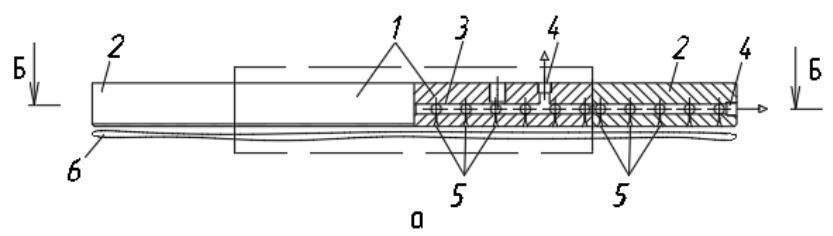

6-6
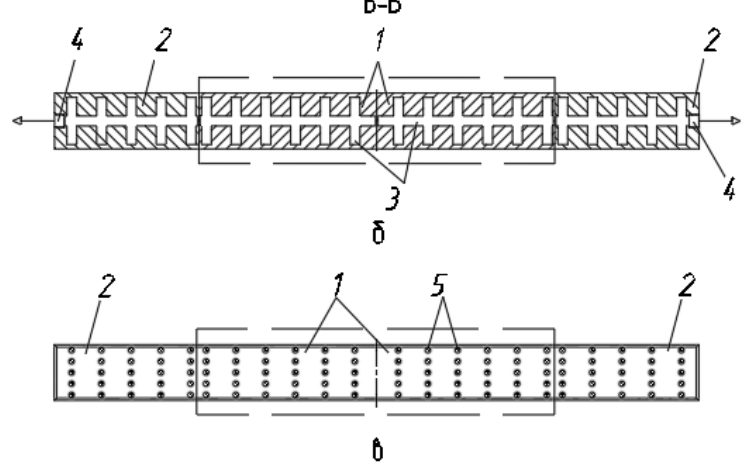

Fig. 1. Design of the modular vacuum gripping device: $a$ - the main view, $b$ - the cut $B-B, c-$ the bottom view (on the working surface side).

Each module represents a housing, with a system of internally branched channels 3 connected to a vacuum source (not shown) through the openings 4 , with a possibility of each section connected to a vacuum source independently. The system of internally branched channels 3 is opened by vacuum chambers 5 on the working (lower) surface of the modules, made in the form of truncated cones, with their large base facing the working surface and located in structured or random order throughout the working surface of the vacuum gripper.

Herewith, the summary linear dimensions of the central modules 1 of the vacuum gripper of the gripping mechanism correspond to the dimensions of the vacuum gripping of the opening mechanism, designed in the same way (shown in dotted lines in the Fig. 1) and are equal to no less than half the width of the flexible container 6 . The dimensions of outer gripping modules 2 of the gripper mechanism are selected in such a way that the length of the working surface of the entire vacuum gripper is equal to the width of the neck of the closed flexible container 6 . 


\subsection{Computer modelling}

To evaluate the working capacity of the vacuum gripping device, a 3D model was designed and its operating principle was modeled using the Solid Works software package (Flow simulation library). The following assumptions have been made during creation of a computer model: absence of flexibility of the container in the area to be gripped and equal roughness of the surface of flexible container. Further, based on the assumptions made, coefficient of reduction in carrying capacity has been corrected.

To simulate air flows in vacuum gripper, required initial and boundary conditions have been set, type of analysis and precision of measurements have been selected. As a result, curves of distribution of air pressure on the circumference of vacuum gripper have been built (Fig. 2).

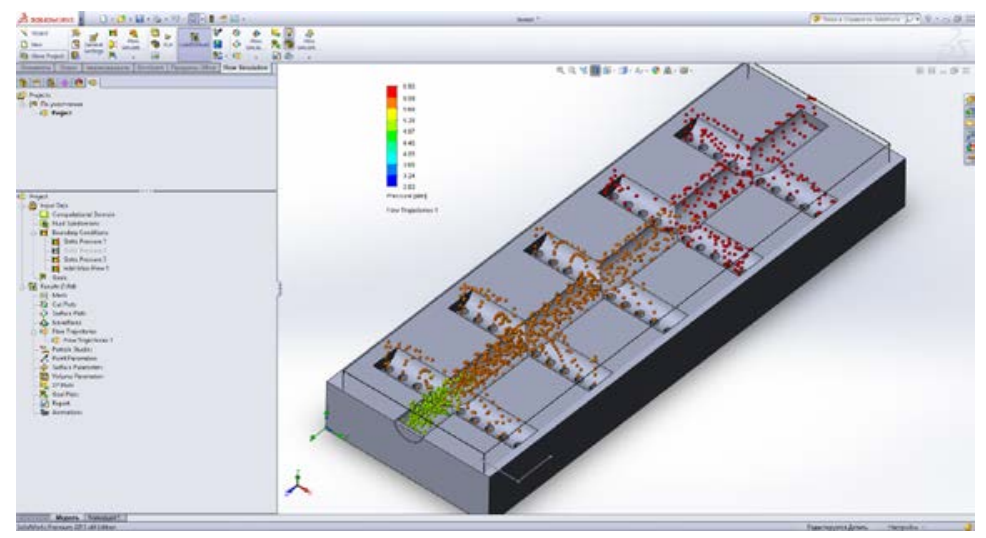

Fig. 2. Curve of the distribution of air pressure on the circumference of vacuum gripper.

From the diagram of the pressure distribution, it is visible that there is sufficient degree of vacuum created inside vacuum gripping device, at the same time distribution on the entire volume of GD is relatively uniform. The analysis of simulation results show the operability of the proposed design and the correct calculation of parameters of VGD.

\subsection{Description of the experimental installation}

One way of making such vacuum grippers may be additive technologies. These technologies facilitate manufacturing of experimental and research samples, and, in connection with the availability of materials and 3D printers [8-9], make it possible to produce prototypes of new devices in a short time, to finalize or optimize existing ones during research, while obtaining sufficient accuracy of products at a low production cost.

Using a 3D printer with 3 degrees of mobility of the STE-320 extruder of the "Stereotec" company, prototypes of a vacuum gripping device consisting of two and four sections were produced (Fig. 3). 


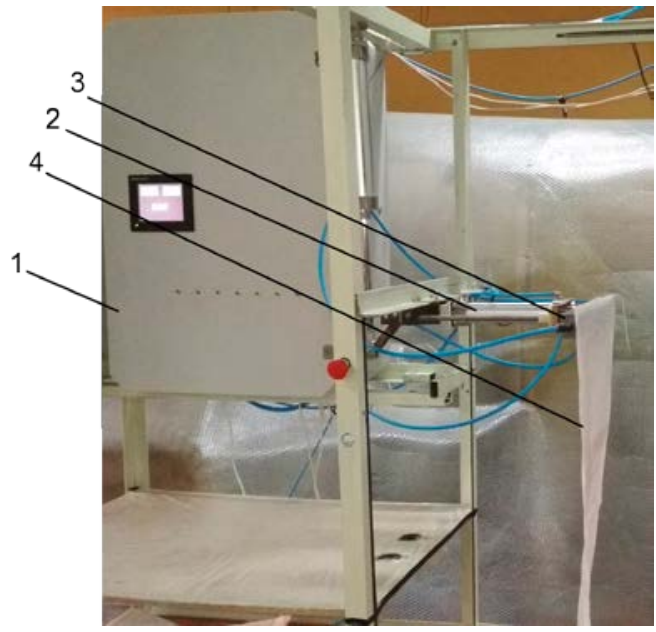

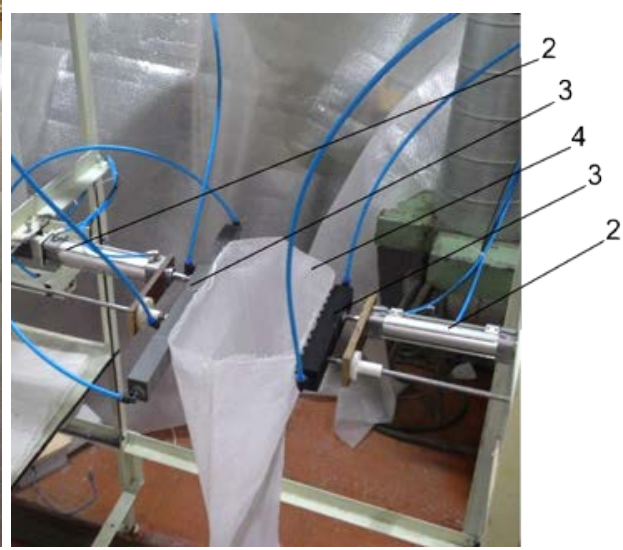

b

Fig. 3. The prototype of a modular VGD produced by the additive technology method: a) FC is captured by all sections, b) FC is captured by the central sections, 1 - control cabinet, 2 - pneumatic cylinder, 3 - VGD, 4 - flexible container.

Control system of the installation is based on PLK FX 3U by Mitsubishi Electric and pneumatic equipment by Camozzi. Microcontroller Arduino UNO is used for automation of the conduction of experiment and processing of results, which is connected to PLK through adapting circuit board based on transistor logic, transforming signals from the outputs of microcontroller $5 \mathrm{~V}$ into the signals with the voltage of $24 \mathrm{~V}$, transferred to PLK that controls electric pneumatic distributors, displacing pneumatic cylinders with VGD and switching vacuum on different VGDs. Ultrasound sensors that measures the distance to the flexible container allow to judge whether it was gripped and opened successfully or not. Vacuum machine creates necessary vacuum the degree of which is measured with pressure sensor connected to the microcontroller and is displayed on vacuum meter for visual control. The degree of vacuum is regulated by servomotor, which conducts the tilt of throttle of regulator to a given degree. Information about current level of vacuum, general quantity of conducted experiments and the number of successful ones is shown on the display. Information from the microcontroller is transmitted to personal computer through a USB port (virtual COM-port).

A program is uploaded to the microcontroller that allows to conduct series of experiments with 100 tests for different degrees of vacuum in automatic mode. Following the launch of the program by pressing button "Launch" a signal is sent from the controller to the servomotor, which rotates, opening regulator that connects the vacuum machine with the vacuum gripping device. After conducting a series of 100 tests, the servomotor interacts with the regulator increasing the degree of vacuum, and the series of tests are repeated.

If the probability of gripping is $100 \%$ (all gripping is successful) after conclusion of the series of tests, one more series is conducted and the trial is concluded. Results of the tests are transferred to a computer for further analysis in Microsoft Excel.

\section{Results and discussion}

Preliminary tests of manufactured samples of modular VGDs were carried out. All samples had vacuum chambers with conical cross-section and working diameter of $2 \mathrm{~mm}$ (outer diameter of the chamber). Earlier research was performed using vacuum suction cups with 
diameters of 30, 40 and $50 \mathrm{~mm}$, the results are described in [10]. Fig. 4 shows the results of an experiment using vacuum grippers in the form of vacuum grippers with a diameter of 30 and $50 \mathrm{~mm}$ with internal dimensions of the vacuum chambers 11 and $20 \mathrm{~mm}$, respectively, and a modular gripping device with chambers $2 \mathrm{~mm}$ in diameter. Reliable gripping of FC is achieved at the degree of underpressure of $-20 \mathrm{kPa}$ (with a diameter of vacuum chambers of $11 \mathrm{~mm})$ and $-22 \mathrm{kPa}(20 \mathrm{~mm})$ with vacuum suction cups, and at $-16 \mathrm{kPa}(2 \mathrm{~mm})$ with modular grippers.

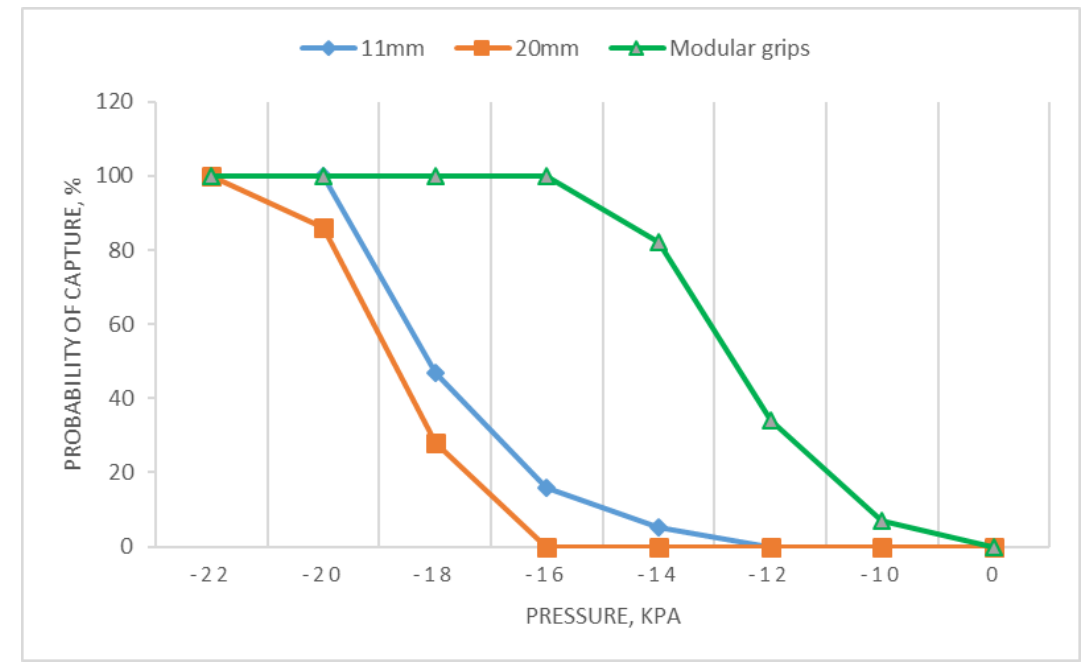

Fig. 4. Results of experiments of opening a flexible container by vacuum grippers with $11 \mathrm{~mm}, 20$ $\mathrm{mm}$ chambers and modular VGD with a chamber diameter of $2 \mathrm{~mm}$.

Based on the results of the experiment, it was established that the quality and probability of successful gripping and holding of FC increases with decrease in diameter and the increase in a number of vacuum chambers, which correlates with increase in speed of lowering of carrying capacity of VGD caused by leakage of air through air-penetrating material of FC and faster underpressurizing. At the same time, increase in the number of vacuum suction cups allows to increase holding force only in the case if vacuum machine allows for required consumption.

The best result was shown by a prototype with vacuum chambers protruding from the gripping area and their random arrangement, because weaved structure of FC has a linear arrangement of pores.

Preliminary tests of the manufactured prototype confirmed the operability of this type of VGD, but an important issue remains to carry out experimental research and optimize the parameters of the developed VGD (the number and dimensions of the vacuum chambers, the required degree of vacuum, etc.).

\section{Conclusions}

The use of the proposed device provides a significant increase in efficiency of holding and manipulating of flexible container from air-penetrating material during filling in comparison with the existing ones. This is provided by the possibility to independently engage different parts of the vacuum gripper of the gripper mechanism and the opening mechanism, creating the necessary vacuum in the vacuum chambers of these grippers. Thus, the gripping and holding of the neck of flexible container occurs over its entire width during gripping and transportation to the loading nozzle, which prevents the flexible 
container from accidentally opening during transport and improves the reliability of holding. Disconnection of the vacuum chambers of outer sections of the vacuum grippers of gripping mechanism from the vacuum source and simultaneous connection to the vacuum source of chambers of the vacuum gripping of the opening mechanism, located as a mirror image, allow to effectively open and securely hold the flexible container evenly over the entire area of its neck from side of the gripping and opening mechanisms during filling flexible container and its subsequent closing for stitching.

Therefore, the use of the given device increases the effectiveness of holding and manipulating a flexible container from air-penetrating material during filling and creates conditions for the complex automation of the packaging process of bulk materials into flexible containers.

Research has been performed with support of the grant of the President of the Russian Federation for young Russian scientists - candidates of science MK-2619.2017.8.

\section{References}

1. A.M. Makarov, Y.P. Serdobincev, Avtomaticheskaya rasfasovka sypuchikh materialov v myagkuyu taru. Povyshenie effektivnosti protsessa [Automatic packaging of bulk materials in flexible container. Increase of process efficiency], Saarbrucken: LAP LAMBERT AcademicPublishing, p.128 (2013)

2. A.M. Makarov, Y.P. Serdobincev, A.S. Sergeev, Research of device for automatic filling of flexible containers with bulk material, Izvestia VSTU. Ser. «Advanced Technology in Machine building». Vol. 10 : Interuniversity Coll. of Scient. Art / VSTU. - Volgograd, № 20 (123), p. 114-117 (2013)

3. A.M. Makarov, Y.P. Serdobincev, E.G. Krilov, Automated filling of flexible containers by bulk products, STIN, № 4, p. 37-40. (2014)

4. A.M. Makarov, Y.P. Serdobincev, O.V. Mushkin, M.A. Lapikov, Mathematical and Computer Modeling of Automated Gripping of Flexible Container with Help of Vacuum, Izvestia VSTU. Ser. «Advanced Technology in Machine building», Volgograd, № 14 (193), p. 63-66 (2016)

5. P. m. 155000 RF IPC B65B5 / 00, B65B7 / 02. The device for the automatic opening, holding and closing the flexible container / A.M. Makarov, M.G. Kristal, A.A. Kovalev, D.A. Suleymanov; VSTU (2015)

6. P. m. 1623937 RF IPC B66C1/02. Vacuum gripping device / V.Y. Krasnoslobodcev, B.B. Brosalin; Leningrad Polytechnic Institute M.I. Kalinina (1988)

7. Patent 2014/0062112 USA, IPC B25J15/06. Vacuum Capture Device / Ho Yang Cho; Korea Pneumatic Systems (2014)

8. A.A. Shvets, A.R. Avdeev, A.V. Drobotov, Comparative analysis of devices for volumetric printing, Izvestia VSTU. Ser. «Advanced Technology in Machine building». - Volgograd, № 1 (156), p. 76-79 (2015)

9. Y.P. Serdobincev, A.V. Drobotov, N.V. Gavrilina, A.R. Avdeev, A.A. Shvets, I.A. Gushin, Method of selecting a 3D printer based on technical and economic indicators, Russian Engineering Research, № 4, p. 37-40 (2017)

10. A.M. Makarov, Y.P. Serdobincev, O.V. Mushkin, M.A. Lapikov, Research of the process of automatic gripping of air-penetrating flexible containers with vacuum during sorting of free-flowing products, Industrial Engineering, Applications and Manufacturing (ICIEAM 2017) (Saint Petersburg, Russia, 16-19 May 2017) : International Conference: Proceedings / St. Petersburg Polytechnic University of Peter the Great, IEEE (Institute of Electrical and Electronics Engineers). - [Publisher: IEEE], 2017, 5 p., DOI: 10.1109/ICIEAM.2017.8076191 